\title{
EL ÁREA FOTOSINTÉTICA COMO INDICADOR DE LA PRODUCCIÓN DE FLORES EN LEPANTHES SANGUINEA
}

\author{
María M. Agosto Pedroza \& Raymond L. Tremblay ${ }^{1}$ \\ Universidad de Puerto Rico - Humacao, Departamento de Biología - 100 carr. 908, Humacao, Puerto Rico 00791. \\ 'Autor para correspondencia: raymond@ hpcf.upr.edu
}

En general, la reproducción en las orquídeas está limitada por el acceso a los polinizadores; (Tremblay, Ackerman, Zimmerman \& Calvo, en revisión); no obstante, la reproducción en la mayoría de las plantas está limitada por el acceso a recursos. Hay algunos ejemplos de limitación por recursos en orquídeas; así en la orquídea Myrmecophilia tibici nis las plantas que son "fertilizadas" por desechos de hormigas realizan un mayor esfuerzo reproductivo (Rico-Gray et al.,1989). Además, Mattila (2000) observó que la producción de frutos en Platanthera bifolia está limitada por el acceso al agua. Pero, en general, cuando se observa limitación por recursos en orquídeas el efecto no es inmediato, sino a largo plazo (múltiples años). El efecto de reducir los recursos a las plantas es una reducción en producción de frutos, flores y crecimiento en los años subsiguientes, no de inmediato (Montalvo \& Ackerman 1987, Zimmerman \& Aide 1989, Calvo 1990, Snow \& Whigham 1989, Primack \& Hall 1990). El acceso a luz para fotosíntesis podría ser uno de los factores limitantes para la reproducción en orquídeas. El área fotosintética puede ser un factor importante en la determinación de la presencia de flores en una planta. En este trabajo determinamos el área fotosintética en treinta plantas de la orquídea Lepanthes san guinea y evaluamos su relación con el esfuerzo reproductivo.

Para esta investigación se utilizaron treinta plantas de Lepanthes sanguinea Hook. Ésta se encuentra distribuida desde la Sierra de Cayey hasta las Montañas de Luquillo en Puerto Rico (Ackerman 1995). Las plantas se estudiaron ex situ creciendo en invernaderos, donde las temperaturas varían entre 21 y $27 \mathrm{C}^{\circ}$ y la humedad entre $70 \%$ y $95 \%$.

El área fotosintética se midió con un papel milimetrado $\left(\mathrm{mm}^{2}\right)$ en forma de transparencia. A cada planta se le sumó el área fotosintética de todas las hojas para obtener el área fotosintética total. Por medio de esto pudimos comparar el área fotosintética de las hojas con la producción total de flores de cada planta, para determinar si existe una correlación. Las hojas con evidencia de inflorescencias (verdes o secas) se consideraron como hojas con flores. Para determinar si hay diferencia en el área fotosintética entre hojas con flores y hojas sin flores se utilizó una prueba de t- no pareada (Zar 1999). En esta prueba, el área fotosintética total fue convertida a logaritmo del área para reducir la heterocedasticidad y normalizar los datos. Se hizo una regresión lineal para comprobar si hay una correlación entre el número de hojas con flores y el área total de hojas.

El área fotosintética está directamante relacionada con la producción de flores. El área mínima de hojas con flores es $60.0 \mathrm{~mm}^{2}$. Los resultados muestran un área promedio de $2.569 \mathrm{~mm}^{2}$ para las hojas con flores y de $1.897 \mathrm{~mm}^{2}$ para las hojas sin flores. El área fotosintética de las hojas con flores es en promedio $35 \%$ mayor que el área de las hojas que no producen flores (t-no pareada, $\mathrm{p}<0.0001$ ).

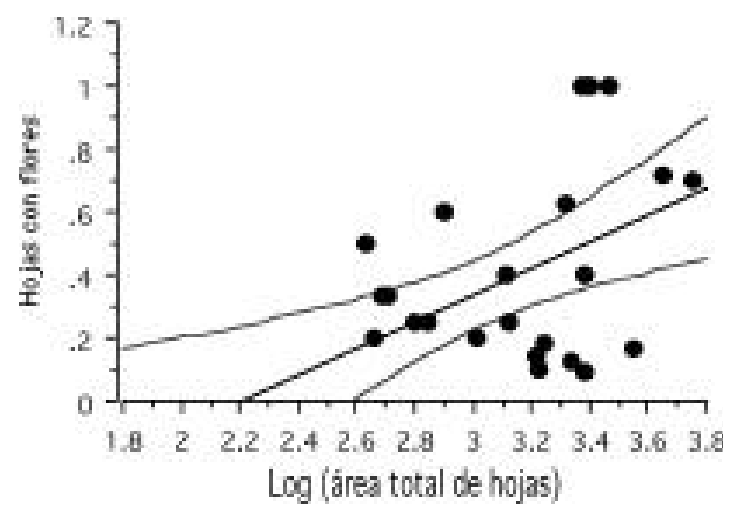

Figura 1. Correlación entre el área fotosintética y el número de flores producidas en Lepanthes sanguinea, y $=-0.939+0.425 \mathrm{x} ; \mathrm{r}^{2}=0.30 ; \mathrm{p}<0.002$. 
La regresión lineal muestra una correlación significativa y positiva entre el área fotosintética total de la planta y el área fotosintética con flores $\left(\mathrm{S}^{2}=0.967, \mathrm{df}=\right.$ $1, \mathrm{P}<0.002)$. La regresión lineal explica solamente parte de la varianza $\left(r^{2}=0.30\right)$. Según estas pruebas, el área fotosintética parece estar directamente relacionada con la presencia de flores y es un buen indicador del esfuerzo reproductivo de la orquídea.

En general, la estructura más importante para la productividad de una planta son las hojas (Ticha 1985). El área fotosintética parece ser un buen indicador de la presencia de flores de la L. sanguinea. Stephenson (1981) encontró que las plantas con hojas grandes tienen una superficie fotosintética mayor y, por tanto, tienen una gran capacidad de relocalizar energía para la reproducción. En esta investigación pudimos constatar que las plantas con mayor área fotosintética tenían mayor número de flores. Alguna plantas tienen más hojas por área fotosintética que otras; esto puede deberse a diferencias estacionales (Kindlmann \& Balounová 1999), estrés ambiental (Primack et al. 1994) o a diferencias genéticas. Si el área fotosintética es menor, esto podría causar un efecto en la calidad de las flores y menor duración de vida de hojas o plantas (Primack et al. 1994). O sea, la disponibilidad de recursos, en este caso la luz para fotosintetizar y el tamaño de área que la recibe, es uno de los factores que indican que la reproducción está limitada por recursos. En otras especies de Lepanthes la producción de frutos y la remoción de polinios en poblaciones naturales está directamente correlacionada con la producción de flores (ver Fernández et al. 2003). Esto significa que el tamaño de la hojas no es el único factor indicador de la presencia de flores o de producción de frutos, ya que puede haber otros factores o recursos que afectan la presencia de flores en Lepanthes sanguinea, como son nitrógeno y fosfato, dos elementos limitantes en el crecimiento de las plantas.

\section{Literatura CitAdA}

Ackerman, J.D. 1995. Orchids of Puerto Rico and the
Virgin Islands. The New York Botanical Garden, Bronx, New York.

Calvo, R.N. 1990. Inflorescence size and fruit distribution among individuals of three orchid species. Amer. J. Bot. 77: 1378-1381.

Fernández, D. S., R. L. Tremblay, J. D. Ackerman, E. Rodriguez \& L.-N. López. 2003. Reproductive potential, growth rate and light environment in Lepanthes rupestris Stimson. Lankesteriana 7: 75-78.

Kindlmann, P. \& Z. Balounová. 1999. Energy partitioning in terrestrial orchids - a model for assessing their performance. Ecol. Model. 119: 167-176.

Mattila, E. 2000. The effect of water stress and pollen availability on reproductive success of Platanthers bifolia (Orchidaceae). Avhandlinger utgitt av Det Norske Videnskaps-Akademi i Oslo. I. MatematiskNaturvidenskapelig Klasse 39: 83-90.

Montalvo, A.M. \& J.D. Ackerman. 1987. Limitation to fruit production in Ionopsis utricularioides. Biotropica 19: 24-31.

Primack, R.B. \& P. Hall 1990. Cost of reproduction in the Pink Lady's slipper orchid: a four year experimental study. Amer. Nat. 136: 638-656.

Primack, R. B., S.L. Miao \& K. R. Becker. 1994. Cost of reproduction in the Pink Lady's slipper orchid (Cypripedium acaule): defoliation, increased fruit production and fire. Amer. J. Bot. 81: 1083-1090.

Rico-Gray, V., J.T. Barber, L.B. Thien, E.G. Ellgaard \& J.J. Toney. 1989. An unusual animal-plant interaction: feeding of Schomburgkia tibicinis (Orchidaceae) by ants. Amer. J. Bot. 76: 603-608.

Snow, A.A. \& D.F. Whigham. 1989. Cost of flower and fruit production in Tipularia discolor. Ecology 70: 1286-1293.

Stephenson, A.G. 1981. Flower and fruit abortion: proximate causes and ultimate funtions. Ann. Rev. Ecol. Syst. 12: 253-279.

Ticha, I. 1985. Ontogeny of leaf morphology and anatomy. In Z. Sestak (ed.), Photosynthesis during leaf development. W. Junk, Dordrecht. pp. 11-15.

Zar, J. 1999. Biostatistical Analysis. Prentice Hall, Inc. 4th edition, New Jersey.

Zimmerman, J.K. \& T.M. Aide. 1989. Patterns of fruit production in a neotropical orchid: pollinator vs. resource limitation. Amer. J. Bot. 76: 67-73.

María M. Agosto Pedroza es estudiante de quinto año de Bachillerato en Biología con concentración en Manejo de Vida Silvestre, de la Universidad de Puerto Rico en Humacao. Esta es su primera experiencia en investigación. Sus aspiraciones futuras son continuar estudios graduados en biología, específicamente en el área de conservación. 